Vol. 52 (1995) [201-206]

\title{
APPROXIMATE POLYHEDRA, DENSITY AND DISCRETE MAPS
}

\author{
antonio Giraldo and Jose M.R. Sanjurjo
}

\begin{abstract}
Some extension properties of maps defined on dense subsets are studied for approximate polyhedra. The latter are characterised as approximate extensors for finite maps with small oscillation.
\end{abstract}

The classes of Approximate Absolute Neighborhood Retracts introduced by Noguchi [12] and Clapp [5] have been widely studied in the literature of theory of retracts. These spaces enjoy important properties connected with Lefschetz's fixed point theorem [5] and [8] and, as Dydak and Segal remark in [6], Clapp's AANR's form a natural class in the sense that they agree with limits of polyhedra in Borsuk's metric of continuity [2].

Moreover, they play an intriguing role in shape theory, in particular in connection with the properties of movability and regular movability $[1,6]$. Clapp's AANR's were recognised by Mardešić as approximate polyhedra $[10,11]$.

The aim of this note is to show that Clapp's AANR's have approximate extension properties for maps defined on dense subsets. These properties are characteristic. In

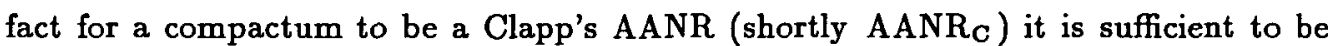
an approximate extensor for finite maps with small oscillation. This result suggests the possibility of developing shape theory and other global theories in Topology in terms of simple internal notions based on dense parts of compacta, in contrast with dual approaches based on neighbourhoods of compacta in suitable ambient spaces (like the Hilbert cube) or more complex intrinsic characterisations, which require the use of multivalued maps $[4,13,14]$. This possibility has actually beeen carried out by the authors in [7]. We recommend the classical books [3] and [9] by Borsuk and Hu respectively for basic information about the theory of retracts. For a more modern approach see Van Mill [15] and for a collection of open problems see West [16].

Let $X$ and $Y$ be metric spaces and let $D$ be a dense subset of $X$. Let $f: D \longrightarrow Y$ be a (not necessarily continuous) function. Given $x \in X$, the oscillation of $f$ at $x$ (possibly infinite) is defined by

$$
O(f, x)=\inf \{\operatorname{diam}(f(U \cap D)) \mid U \text { a neighbourhood of } x \text { in } X\} .
$$

Received 2nd November, 1995.

The second author is partially supported by DGICYT.

Copyright Clearance Centre, Inc. Serial-fee code: 0004-9729/95 \$A2.00+0.00. 
By a map we always understand a continuous function. We say that a map $f: D \longrightarrow Y$ is discrete if the set $f(D)$ is finite. (Observe that if $f$ is a discrete map then it is locally constant).

APPRoximation Lemma. Suppose $Z$ is a normed vector space or, more generally, a convex subset of a normed vector space. Let $X$ be a metric space and $Y$ a subset of $Z$. Let

$$
f: X \longrightarrow B_{\varepsilon}(Y)=\{z \in Z \mid d(z, Y)<\varepsilon\}
$$

be a map. Then there exists an open dense subset $D$ of $X$ and there exists a locally constant map $g: D \longrightarrow Y$ such that $O(g, x) \leqslant 2 \varepsilon$ for every $x \in X$ and such that $d(f(x), g(x))<\varepsilon$ for every $x \in D$.

Conversely, given a dense subset $D$ of $X$ and given a function $f: D \longrightarrow Y$ with $O(f, x)<\varepsilon$ for every $x \in X$, there exists a map $g: X \longrightarrow \overline{B_{\varepsilon}(Y)}$ such that $d(f(x), g(x))<\varepsilon$ for every $x \in D$.

Proof: Consider the set $G$ of all the locally constant maps $g: G_{g} \longrightarrow Y$ defined on open subsets $G_{g}$ of $X$ and such that $d(f(x), g(x))<\varepsilon$ for every $x \in G_{g}$.

Then $G$ is not empty. Given $x_{0} \in X$ there exists $y_{0} \in Y$ such that $d\left(f\left(x_{0}\right), y_{0}\right)<\varepsilon$ and by the continuity of $f$ there exists an open neighbourhood $U$ of $x_{0}$ in $X$ such that for every $x \in U$ we have $d\left(f(x), f\left(x_{0}\right)\right)<\varepsilon-d\left(f\left(x_{0}\right), y_{0}\right)$ and hence $d\left(f(x), y_{0}\right)<\varepsilon$. Thus the function $h: U \longrightarrow Y$ given by $h(x)=y_{0}$, for every $x \in U$, is an element of $G$.

We now consider in $G$ the following order relation:

$$
g \leqslant h \Leftrightarrow G_{g} \subset G_{h} \text { and }\left.h\right|_{G_{g}}=g .
$$

$(G, \leqslant)$ is an ordered set and every totally ordered subset of $G$ has an upper bound (if $A \subset G$ is a totally ordered subset of $G$, then $G_{h}=\bigcup_{g \in A} G_{g}$ is an open subset of $X$ and the function $h: G_{h} \longrightarrow Y$ given by $h(x)=g(x)$ for any $g \in A$ with $x \in G_{g}$ is a well defined locally constant map and is an upper bound for $A$ ). Then we can apply Zorn's Lemma and deduce the existence of a maximal element.

Let $g: D \longrightarrow Y$ be one of these maximal elements and suppose that $D$ is not dense. Then there exists an open subset $B$ of $X$ such that $B \cap D=\emptyset$. Given $x_{0} \in B$, there exists $y_{0} \in Y$ such that $d\left(f\left(x_{0}\right), y_{0}\right)<\varepsilon$ and by the continuity of $f$ there exists an open neighbourhood $U \subset B$ of $x_{0}$ in $X$ such that for every $x \in U$ we have $d\left(f(x), f\left(x_{0}\right)\right)<\varepsilon-d\left(f\left(x_{0}\right), y_{0}\right)$ and hence $d\left(f(x), y_{0}\right)<\varepsilon$. But then the function $h: D \cup U \longrightarrow Y$ given by

$$
h(x)= \begin{cases}g(x) & \text { if } x \in D \\ y_{0} & \text { if } x \in U\end{cases}
$$


is locally constant, is defined on an open subset of $X$ and $d(f(x), h(x))<\varepsilon$ for every $x \in D \cup U$, and this is in contradiction with the maximality of $g$. Therefore, $D$ has to be dense.

Finally, since $d(f(x), g(x))<\varepsilon$ for every $x \in D$ and $f$ is continuous in $X$, then $O(g, x) \leqslant 2 \varepsilon$ for every $x \in X$.

Let us prove the second part of the Lemma. Suppose given $f: D \longrightarrow Y$ such that $D$ is a dense subset of $X$ and such that $O(f, x)<\varepsilon$ for every $x \in X$. Then for every $x \in X$ there exists an open neighbourhood $U_{x}$ of $x$ in $X$ such that $\operatorname{diam}\left(f\left(U_{x} \cap D\right)\right)<$ $\varepsilon$. Since $X$ is a metric space, it is paracompact and hence there exists a locally finite open covering $\left\{U_{i} \mid i \in I\right\}$ of $X$ such that $\operatorname{diam}\left(f\left(U_{i} \cap D\right)\right)<\varepsilon$ for every $i \in I$.

We define, for every $i \in I, \lambda_{i}: X \longrightarrow \mathbb{R}$ such that

$$
\lambda_{i}(x)=\frac{d\left(x, X-U_{i}\right)}{\sum_{j \in I} d\left(x, X-U_{j}\right)} .
$$

The sum in the denominator is finite since $d\left(x, X-U_{j}\right) \neq 0$ if and only if $x \in U_{j}$. Moreover for every $x \in X$,

$$
\sum_{i \in I} \lambda_{i}(x)=1
$$

and hence, for every $x \in X$ we have

$$
f(x)=\sum_{i \in I} \lambda_{i}(x) f(x)
$$

We now choose, for every $i \in I$, a point $x_{i} \in U_{i} \cap D$ and define $g: X \longrightarrow Z$ by the expression

$$
g(x)=\sum_{i \in I} \lambda_{i}(x) f\left(x_{i}\right) .
$$

This is again a finite sum and, since $\sum \lambda_{i}(x)=1$ and $Z$ is convex, $g$ is a well defined continuous function. Moreover, if for every $x \in D$ we consider the open sets $U_{j_{1}}, \ldots, U_{j_{n}}$ to which $x$ belongs, then we have

$$
\begin{aligned}
d(f(x), g(x)) & =\left\|\sum_{i=1}^{n} \lambda_{j_{i}}(x) f(x)-\sum_{i=1}^{n} \lambda_{j_{i}}(x) f\left(x_{j_{i}}\right)\right\|=\left\|\sum_{i=1}^{n} \lambda_{j_{i}}(x)\left(f(x)-f\left(x_{j_{i}}\right)\right)\right\| \\
& \leqslant=\sum_{i=1}^{n} \lambda_{j_{i}}(x)\left\|f(x)-f\left(x_{j_{i}}\right)\right\|<\varepsilon\left(\sum_{i=1}^{n} \lambda_{j_{i}}(x)\right)=\varepsilon
\end{aligned}
$$

Therefore $d(f(x), g(x))<\varepsilon$ for every $x \in D$. (In the above expressions we have used the norm \|| $\|$ of the normed vector space in which $Y$ is contained). 
Finally, since $g$ is continuous in $X$ and $g(D) \subset B_{\varepsilon}(Y)$ with $D$ dense in $X$, then $g(X) \subset \overline{B_{\varepsilon}(Y)}$.

Definition: A compact metric space $X$ is an AANR $\mathrm{A}_{\mathrm{C}}$ provided when $X$ is embedded in the Hilbert cube $Q$, then for every $\varepsilon>0$ there exists a neighbourhood $U$ of $X$ in $Q$ and there exists a map $r_{\varepsilon}: U \longrightarrow X$ such that $d\left(r_{\varepsilon}(x), x\right)<\varepsilon$ for every $x \in X$.

We introduce in the next definition the notion of approximate polyhedron. By a result of Mardešić [10], approximate polyhedra agree with Clapp's AANR's in the category of compact metric spaces.

Definition: A metric space $X$ is an approximate polyhedron if for each $\varepsilon>0$ there is a polyhedron $P$ and maps $f: X \longrightarrow P, g: P \longrightarrow X$ such that the distance $d(g f(x), x)<\varepsilon$ for all $x \in X$.

In the following theorem we denote by

$$
O(f, X)=\sup _{x \in X} O(f, x)
$$

the oscillation of $f$ in $X$.

THEOREM. Let $Y$ be a compact metric space. Then the following statements are equivalent:

(1) $Y$ is an AANR $_{C}$.

(2) For every $\varepsilon>0$ there exists $\delta>0$ with the property that for every metric space $X$, every dense subset $D$ of $X$ and every function $f: D \longrightarrow Y$ with $O(f, X)<\delta$, there exists a continuous function $f^{\prime}: X \longrightarrow Y$ such that $d\left(\left.f^{\prime}\right|_{D}, f\right)<\varepsilon$.

(3) For every $\varepsilon>0$ there exists $\delta>0$, with the following property (*):

For every compact metric space $X$, every open dense subset $D$ of $X$ and every discrete map $f: D \longrightarrow Y$ with $O(f, X)<\delta$, there exists a continuous function $f^{\prime}: X \longrightarrow Y$ such that $d\left(\left.f^{\prime}\right|_{D}, f\right)<\varepsilon$.

Proof: We prove first that (1) implies (2). Let $Y$ be a compact metric space. We can assume that $Y$ lies in the Hilbert cube, $Q$. Suppose that $Y$ is an AANR $_{\mathrm{C}}$. Then for every $\varepsilon>0$, there exists a compact neighbourhood $U$ of $Y$ in $Q$ and there exists a map $r_{\varepsilon}: U \longrightarrow Y$, such that $d\left(r_{\varepsilon}(y), y\right)<\varepsilon / 3$ for every $y \in Y$. Since $U$ is compact and $r_{e}$ is continuous, there exists $\delta>0$ such that

$$
d\left(r_{\varepsilon}(y), r_{\varepsilon}\left(y^{\prime}\right)\right)<\frac{\varepsilon}{3}
$$

for every $y, y^{\prime} \in U$ with $d\left(y, y^{\prime}\right)<\delta$. We can suppose that $\delta$ also satisfies that $\overline{B_{\delta}(Y)} \subset U$. 
Consider now a metric space $X$ and let $D$ be a dense subset of $X$. Let $f: D \longrightarrow Y$ be a function with $O(f, X)<\delta$. Then $O(f, x)<\delta$ for every $x \in X$ and, by the Lemma, there exists a continuous function $f^{\prime \prime}: X \longrightarrow \overline{B_{6}(Y)} \subset U$ such that $d\left(f^{\prime \prime}(x), f(x)\right)<\delta$, for every $x \in D$. Consider $f^{\prime}=r_{e} f^{\prime \prime}: X \longrightarrow Y$. Then $f^{\prime}$ is a continuous function and for every $x \in D$, since $d\left(f^{\prime \prime}(x), f(x)\right)<\delta$, we have that

$$
d\left(f^{\prime}(x), f(x)\right) \leqslant d\left(r_{\varepsilon} f^{\prime \prime}(x), r_{e} f(x)\right)+d\left(r_{e} f(x), f(x)\right)<\frac{2 \varepsilon}{3} .
$$

Therefore $d\left(\left.f^{\prime}\right|_{D}, f\right)<\varepsilon$.

Obviously (2) implies (3). To prove that (3) implies (1) we assume again that $Y$ lies in $Q$. Given $\varepsilon>0$ consider $0<\delta<(2 \varepsilon) / 3$ satisfying $(*)$ for $\varepsilon / 3$. Consider the compact neighbourhood $\overline{B_{\delta / 4}(Y)}$ of $Y$ in $Q$ and consider the identity map

$$
i: \overline{B_{\delta / 4}(Y)} \rightarrow \overline{B_{\delta / 4 z}(Y)} .
$$

Since $Y$ is compact, there exists a finite subset $F$ of $Y$ such that $\overline{B_{\delta / 4}(Y)} \subset B_{\delta / 3}(F)$. Then, by the Lemma, there exists an open dense subset $D$ of $\overline{B_{\delta / 4}(Y)}$ and there exists a locally constant, and hence discrete, map $f: D \longrightarrow F \subset Y$ such that $d(f(x), x)<\delta / 2$ for every $x \in D$ and such that $O(f, x)<\delta$ for every $x \in \overline{B_{\delta / 4}(Y)}$. Moreover, since $f(D)$ is a finite set, $O(f, x)$ can only take a finite number of different values. This implies that

$$
O\left(f, \overline{B_{\delta / 4}(Y)}\right)=\max _{x \in \overline{B_{\delta / 4}(Y)}} O(f, x)<\delta .
$$

By property (*), there exists a continuous function

$$
r_{e}: \overline{B_{\delta / 4}(Y)} \longrightarrow Y
$$

such that $d\left(\left.r_{\varepsilon}\right|_{D}, f\right)<\varepsilon / 3$. Therefore, for every $x \in D$ we have

$$
d\left(r_{\varepsilon}(x), x\right) \leqslant d\left(r_{\varepsilon}(x), f(x)\right)+d(f(x), x)<\frac{\varepsilon}{3}+\frac{\delta}{2}<\frac{2 \varepsilon}{3} .
$$

Finally, since $r_{\varepsilon}$ is continuous in $\overline{B_{\delta / 4}(Y)}$ and $D$ is dense in $\overline{B_{6 / 4}(Y)}$ we have that

$$
d\left(r_{\varepsilon}(x), x\right) \leqslant \frac{2 \varepsilon}{3}<\varepsilon
$$

for every $x \in \overline{B_{\delta / 4}(Y)}$. 


\section{REFERENCES}

[1] S. Bogatyi, 'Approximative and fundamental retracts', Math. USSR-Sb. 22 (1974), 91-103.

[2] K. Borsuk, 'On some metrizations of the hyperspace of compact sets', Fund. Math. 41 (1954), 168-202.

[3] K. Borsuk, Theory of retracts, Monografie Matematyczne 44 (Polish Scientific Publishers, Warszawa, 1967).

[4] K. Borsuk, 'Concerning homotopy properties of compacta', Fund. Math. 62 (1968), 223-254.

[5] M.H.Clapp, 'On a generalization of absolute neighborhood retracts', Fund. Math. 70 (1971), 117-130.

[6] J. Dydak and J. Segal, 'Approximate polyhedra and shape theory', Topology Proc. 6 (1981), 279-286.

[7] A. Giraldo and J.M.R. Sanjurjo, 'Density and shape', (preprint).

[8] A. Granas, 'Fixed point theorems for the approximative ANRs', Bull. Acad. Polon. Sci., Ser. sci. math., astr. et phys. 16 (1968), 15-19.

[9] S. Hu, Theory of retracts (Wayne State University Press, Detroit, 1967).

[10] S. Mardešić, 'Approximate polyhedra, resolutions of maps and shape fibrations', Fund. Math. 114 (1981), 53-78.

[11] S. Mardešić and J. Segal, Shape theory (North Holland, Amsterdam, 1982).

[12] H. Noguchi, 'A generalization of absolute neighborhood retracts', Kodai Math. Seminar reports 1 (1953), 20-22.

[13] J.M.R. Sanjurjo, 'An intrinsic description of shape', Trans. Amer. Math. Soc. 329 (1992), 625-636.

[14] J.M.R. Sanjurjo, 'Multihomotopy, Cech spaces of loops and shape groups', Proc. London Math. Soc. 69 (1994), 330-344.

[15] J. Van Mill, Infinite-dimensional topology (North Holland, Amsterdam, 1989).

[16] J.E. West, 'Problems in infinite-dimensional topology', in Open problems in topology, (J. Van Mill and G.M. Reed, Editors) (North Holland, Amsterdam, 1990), pp. 523-597.

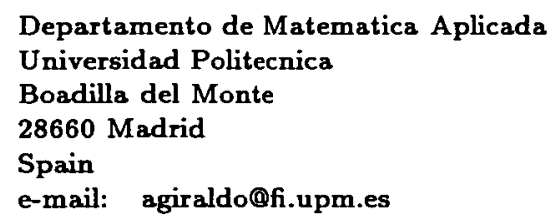

\author{
Departamento de Geometria y Topologia \\ Universidad Complutense \\ 28040 Madrid \\ Spain \\ e-mail: W745@emducm11.sim.ucm.es
}

